\title{
VALIDATION OF A GREEK VERSION OF PSS-14; A GLOBAL MEASURE OF PERCEIVED STRESS
}

\author{
Alexia Katsarou ${ }^{1}$, Demosthenes Panagiotakos ${ }^{2}$, Aggeliki Zafeiropoulou, Marios Vryonis ${ }^{3}$, loannis Skoularigis ${ }^{1}$, \\ Filippos Tryposkiadis ${ }^{1}$, Charalabos Papageorgiou ${ }^{4}$ \\ ${ }^{1}$ Department of Cardiology, School of Medicine, University of Thessaly, Larissa, Greece \\ ${ }^{2}$ Group of Biostatistics and Research Methods, Department of Nutrition and Dietetics, Harokopio University, Athens, Greece

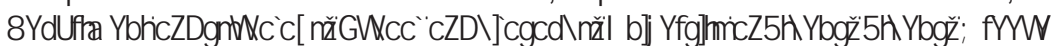 \\ First Department of Psychiatry, Eginition Hospital, School of Medicine, University of Athens, Athens, Greece
}

\section{SUMMARY}

Aim: To evaluate validity of the Greek version of a global measure of perceived stress PSS-14 (Perceived Stress Scale - 14 item).

Materials and Methods: The original PSS-14 (theoretical range 0-56) was translated into Greek and then back-translated. One hundred men and women (39 \pm 10 years old, $40 \mathrm{men}$ ) participated in the validation process. Firstly, participants completed the Greek PSS-14 and, then they were interviewed by a psychologist specializing in stress management. Cronbach's alpha (a) evaluated internal consistency of the measurement, whereas Kendall's tau-b and Bland \& Altman methods assessed consistency with the clinical evaluation. Exploratory and Confirmatory Factor analyses were conducted to reveal hidden factors within the data and to confirm the two-dimensional character of the scale.

Results: Mean (SD) PSS-14 score was 25(7.9). Strong internal consistency (Cronbach's a $=0.847$ ) as well as moderate-to-good concordance between clinical assessment and PSS-14 (Kendall's tau- $b=0.43, p<0.01$ ) were observed. Two factors were extracted. Factor one explained $34.7 \%$ of variability and was heavily laden by positive items, and factor two that explained $10.6 \%$ of the variability by negative items. Confirmatory factor analysis revealed that the model with 2 factors had chi-square equal to 241.23 ( $p<0.001$ ), absolute fix indexes were good (i.e. GFI $=0.733$, $\mathrm{AGFI}=0.529$ ), and incremental fix indexes were also adequate (i.e. $\mathrm{NFI}=0.89$ and $\mathrm{CFI}=0.92$ ).

Conclusion: The developed Greek version of PSS-14 seems to be a valid instrument for the assessment of perceived stress in the Greek adult population living in urban areas; a finding that supports its local use in research settings as an evaluation tool measuring perceived stress, mainly as a risk factor but without diagnostic properties.

Key words: PSS-14, validation, stress, Greece

Address for correspondence: A. L. Katsarou, 6 Giannitsioti St., Larissa, 41222, Greece. E-mail: KatsarouAlexia@gmail.com

\section{INTRODUCTION}

Stress refers to the process of adaptation of an individual once exposed to external or internal challenges (1). Maladaptation to stress alters both brain function and peripheral physiology, and can promote a broad array of psychological (e.g. anxiety, depression, burnout, memory deficits, and pain disorders) and organic disturbances. It has been documented that stress-related conditions account for more than half of all disabilities (2). In this framework it has been estimated that workplace stress results in accidents, employee turnover, diminished productivity, direct medical, legal, and insurance costs consequentially giving rise to a tremendous financial burden as well as stress-related disorders (3). Perceived stress can be viewed as the outcome of transactional interaction between the person and his environment. In other words, cognitive appraisal or subjective evaluation of an individuals' environment has been postulated to be the key process to the stress outcome(s), rather than the single occurrence of an event (4-11).

The continuous increase of stress-related disorders in civilized countries and the rapid growth of knowledge on the psychobiological mechanisms behind them are challenging clinicians and researchers across different disciplines $(12,13)$. In this sense subjective reports provide one source of information which may result in appropriate diagnostic and therapeutic interventions. Several tools (i.e. questionnaires) have been proposed in order to measure psychological stress. The majority of these tools have been tested for their reliability and validity in the referent population as well as other populations. Perceived Stress Scale (PSS) is a valid and reliable instrument in congruent measurement of life stress appraisal among the US population (14). In particular, Cohen and colleagues developed this global measure of stress by asking respondents to evaluate the level to which their lives seem to be unpredictable, uncontrollable or overloaded (14). The PSS is one of the most frequently used tools to measure stress in chronic conditions and situations often not listed in other life-event scales.

The high prevalence of chronic and stress-related diseases, and risk factors that have been reported in Greece (e.g. cardiovascular disease, metabolic syndrome, overweight, obesity, depression) underline importance of the development of a validation tool suitable for the Greek population and culture (15-17). However, use of readily available instruments developed for other populations affords the opportunity (although with some limitations) for intercountry, inter-culture and inter-race comparisons. Thus, the aim of this work was to test validity of a Greek version of PSS-14 on an adult sample from the general population. 


\section{MATERIALS AND METHODS}

\section{The PSS-14}

The original and largest of the three PSS versions (i.e. PSS-14 with 14 items) was selected for translation and validation. PSS-14 is considered as a brief and easy scale to administer and complete. It consists of seven negative and seven positive items, each with a possible answer rated on a five-point scale (from $0=$ never to 4 = very often). The highest possible score is 56 , since positive questions were rated from 4 to 0 and negative questions were rated on the opposite scale. The positive element evaluates the ability to cope with perceived stressors, whereas the negative one focuses on assessing lack of control, negative emotions and reactions.

The translation and back-translation procedure was as follows: the English version of the PSS-14 was firstly translated into Greek by a native speaker and afterwards the Greek item was back translated into English by a native English speaker psychologist. Special care was taken to ensure that each item retained its original meaning. The content and face validity (i.e. the extent to which a measure represents all facets of a given social construct) of PSS-14 was evaluated by a psychologist, a member of the study group, and was in accordance with the original tool.

\section{Participants and Procedure}

The study sample consisted of 100 participants $(39 \pm 10$ years old females and 40 years old males), 60 residents of Athens (Attica Prefecture) and 40 residents of Larissa (Thessaly Prefecture). Both cities comprise large urban areas (Athens is the metropolitan capital of Greece and Larissa the metropolitan hub of Thessaly). These participated in the validation process with a response rate of $95 \%$ (i.e. 100 out of 105 participants that were initially asked to participate, finally agreed). The sample size of 100 participants was a-priori decided in order to achieve statistical power greater than $95 \%$ for the evaluation of two-sided mean differences between the tool and the clinical assessment equal to $5 \pm 2$ at 0.05 type-I error (GPower v. 3.1.0, Kiel University, Germany).

\section{Method of Study Sample Selection}

Recruitment took place mostly at work place. Participants were enrolled on a voluntary basis but were not randomly selected; nonetheless, an effort was made to avoid any systematic selection bias and the selected study sample should be representative of all aspects of the reference population of the two cities. The selection criteria of recruitment included: age over 18 years, and both genders. No exclusion criteria were applied.

The PSS-14 was initially distributed to and completed by all participants and, upon their collection, a psychologist experienced in the field of stress clinically assessed the stress levels of each participant in private. The clinical assessment was based on a structured interview by a study team psychologist, whose aim to retrieve information about stressful situations undergone by the individual interviewee during the previous time to the study month, existence and nature of the subject's coping mechanisms, the extent and effectiveness of his social support network and existence of physical symptoms, if any.
Participants were also asked to report their age, family status (married, never married, divorced, widowed), type of occupation, economic status and the number of children each had; whereas they were also coded as whether they had children or not. In particular, regarding financial status, participants were classified as those satisfied with their income and those who were not. All the information was collected in order to be used in a more in depth analysis of the PSS-14 validation process.

\section{Bioethics}

The retrieved data was confidential and the study followed the ethical considerations provided by the World Medical Association (52nd WMA General Assembly, Edinburg, Scotland, October 2000). All participants were informed of the procedures of the study and agreed to participate providing written informed consent.

\section{Statistical Methods}

Descriptive statistics are presented as mean values $( \pm \mathrm{SD})$ and quartiles. To evaluate the internal consistency of the PSS-14, the Cronbach's alpha was calculated. The computation of Cronbach's alpha was based on the number of items of the survey (i.e. k) and the ratio of the average inter-item covariance to the average item variance. In particular, the formula alpha $=[\mathrm{k} *($ inter-item covariance/average variance $)] /$ $[1+(\mathrm{k}-1) *($ inter-item covariance/average variance $)]$. Kendall tau-b coefficient (the coefficient ranges between -1 and +1 ; values $>0.3$ suggest moderate agreement, while values $>0.6$ suggest good agreement) and the Bland \& Altman method of agreement was used to evaluate the validity of the scale. With respect to the Bland \& Altman method, the limits of agreement were calculated as mean (difference) $\pm 1,96 *$ standard deviation (difference) assuming normal distribution of the differences. Normality was tested using P-P plots. Sub-group (sensitivity) analysis was performed to further evaluate the validity of the PSS-14 for men and women as well as for participants' age class, family status, occupation and economic level. The significance level for all two-sided hypotheses tested was considered at 0.05 . Furthermore, exploratory factor analysis was applied. The principal component method and the correlation matrix of the PSS-14 items were used (but the principal axis factoring was also applied to confirm the results) to extract factors. Factors with eigenvalues greater than 1.0 were retained and interpreted. Factor loadings were calculated after varimax rotation and values $>0.4$ were used to characterize its factor. The Kaiser-Myer-Oklin criterion and the Bartletts's test of sphericity were calculated in order to evaluate the level of inter-correlation of the PSS-14 items. Confirmatory analysis was then applied to test the results revealed from exploratory factor analysis. Chi-square statistic was calculated to test the model's goodness-of-fit, absolute (GFI, AGFI) and incremental (NFI, CFI) goodness of fit indexes were also calculated to evaluate whether the proposed model reproduces the data (values close to 1 indicate adequate fit; this index was preferred since it avoids the underestimation of fit, often noted in small samples. All statistical analyses were performed using the SPSS and AMOS version 18 (SPSS Inc., Chicago, II, USA). 


\section{RESULTS}

The majority of participants worked as public employees $(63 \%)$, whereas others worked in private companies $(22 \%)$, and few $(2 \%)$ were unemployed. Additionally, $63 \%$ reported discontent with their income, $51 \%$ were married and $53 \%$ of participants were parents (Table 1).

The mean $( \pm \mathrm{SD})$ value of the PSS -14 for the whole sample was $25.29 \pm 7.9$, while the quartiles (1st, median and $3 \mathrm{rd}$ ) were 19 , 24 and 31, respectively. Furthermore, the gender-specific distribution of the PSS-14 was in men: (mean $( \pm \mathrm{SD})$ ): $24.39 \pm 8.2$, and in women $25.89 \pm 7.6$, while the quartiles (1st, median and 3rd) were $18.75,24,30.25$ in men and 20, 25, 32 in women, respectively.

\section{Reliability and Validity of the PSS-14}

According to the results described in Table 3, it can be seen that the agreement between clinical assessment and PSS-14 evaluation was good, regarding the whole sample of the study (Kendall's tau-b $=0.43, \mathrm{p}<0.001$ ). Furthermore, a very good internal consistency was revealed $($ Cronbach's alpha $=0.84$ ) for the overall sample (at this point it should be noted that the scaling of items used for evaluation of internal consistency was according to the original direction described by the PSS-14). In addition, when each item of the PSS-14 was excluded from the analysis in order to test the robustness of the findings, Cronbach's alpha remained high (i.e. $>0.80$ ). The Bland \& Altman Method confirmed the aforementioned results showing a satisfactory and acceptable difference (bias) between the clinical assessment and the PSS-14 scores, nonetheless the relatively high limits of agreement were determined. Moreover, for the whole study group the sensitivity of PSS-14 for a threshold equal to 25 was $78 \%$, the specificity was $61 \%$ and the area under the curve was $0.78 \pm 0.05(\mathrm{p}<0.001)$.

Table 1. Descriptive characteristics of the study's sample

\begin{tabular}{|c|c|}
\hline $\mathrm{n}$ & 100 \\
\hline Age (yrs) & $39 \pm 10$ \\
\hline \multicolumn{2}{|l|}{ Gender } \\
\hline Males, $n$ & 40 \\
\hline Females, $\mathrm{n}$ & 60 \\
\hline \multicolumn{2}{|l|}{ Occupation } \\
\hline Free lancers, $n$ & 13 \\
\hline Private employees, $n$ & 22 \\
\hline Public employees, $n$ & 63 \\
\hline Unemployed, $n$ & 2 \\
\hline Participants satisfied with their income, $n$ & 37 \\
\hline \multicolumn{2}{|l|}{ Family status } \\
\hline Non-married, n & 41 \\
\hline Married, $n$ & 51 \\
\hline Divorced, $\mathrm{n}$ & 4 \\
\hline Widowed, $n$ & 4 \\
\hline Having children, $n$ & 53 \\
\hline
\end{tabular}

\section{Exploratory and Confirmatory Factor Analysis}

The KMO value was 0.841 , suggesting very good intercorrelation level among the PSS-14 items, and the sphericity test was significant suggesting that the results are acceptable. As it can be seen in Table 2, two factors were extracted. Factor 1 that explained $34.7 \%$ of variability was heavily encumbered by items that related to coping ability, sense of control in life and personal issues, feeling that things are going right and ability to manage time. Factor 2 explained $10.6 \%$ of variability and was heavily laden by items relating to feelings of distress, weakened control of significant issues in life, weakness to cope with personal duties and time management. The correlation between factor 1 and factor 2 was -0.477 . Confirmatory factor analysis revealed that the model with 2 factors had chi-square equal to $241.23(\mathrm{p}<0.001)$, absolute fix indexes were good (i.e. $\mathrm{GFI}=0.733$, AGFI $=0.529$ ), and incremental fix indexes were also adequate (i.e., NFI $=0.89$ and $\mathrm{CFI}=0.92$ ).

\section{Sensitivity Analysis for the Validity of the PSS-14}

Subgroup analyses showed that Kendall's tau-b coefficients were significant in all sub-groups, by the exception of free-lancers (Table 3). Moreover, all Cronbach's alpha values were greater than 0.8 , with higher values observed in private employees, those who were married, women, under 40 , those who had children and those who were satisfied with their income (the highest value was found for free lancers, Cronbach's alpha $=0.931$ and the lowest for non-married participants, Cronbach's alpha $=0.809$ ). The intra-correlation coefficients (ICC) of the PSS-14 among the subgroups was also measured and found statistically significant in each group (data not shown here).

Sex exploratory analysis revealed similar factors as the whole group (i.e. factor 1 was heavily laden down by coping ability, sense of control, and had $35 \%$ total variance explained; while factor 2 was heavily burdened by items relating to feelings of distress, weakened control on significant issues in life and had $12 \%$ total variance explained). Distribution analysis by family status, income satisfaction, and age-group also revealed the aforementioned two factors in both $<40$ and $>40$ year old participants (data not shown here).

The Bland \& Altman Method revealed that the mean differences (bias) between the two methods for the subgroup analyses ranged from 1.51 to 6.52 . Significantly lower levels of bias were found for those who had no children as compared with those who had ( $\mathrm{p}=0.009$ ), and for those who were $<40$ years old as compared with those over $40(\mathrm{p}=0.006)$ (Table 3$)$.

\section{DISCUSSION}

The aim of this work was to evaluate validity of a perceived stress scale PSS-14 in a Greek sample. For this reason a sample of 100 middle aged individuals from two large cities were enrolled. According to data analysis, the Greek version of PSS-14 seems to be a valid stress instrument (criterion validity) for the studied population. Two factors were revealed here, one with positive (factor 1) and the other with negative items (factor 2). These factors were also observed when the analysis was stratified by sex, age group and marital status. At this point it should be noted 
Table 2. Results from exploratory factor analysis that was applied in the responses of $n=100$ individuals as regards the PSS-14 items

\begin{tabular}{|l|c|c|}
\hline PSS-14 item & Factor 1 & Factor 2 \\
\hline 1. In the last month, how often have you felt anxious for something that happened unexpectedly? & -0.136 & 0.800 \\
\hline 2. In the last month, how often have you felt unable to control the important things in your life? & 0.229 & 0.466 \\
\hline 3. In the last month, how often have you felt nervous and "stressed"? & 0.359 & 0.712 \\
\hline 4. In the last month, how often have you dealt successfully with day to day problems and annoyances? & 0.701 & -0.047 \\
\hline $\begin{array}{l}\text { 5. In the last month, how often have you felt that you were effectively coping with important changes that were occurring in } \\
\text { your life? }\end{array}$ & 0.598 \\
\hline 6. In the last month, how often have you felt confident about your ability to handle your personal problems? & 0.059 \\
\hline 7. In the last month, how often have you felt that things were going your way? & 0.585 & 0.063 \\
\hline 8. In the last month, how often have you found that you could not cope with all the things that you had to do? & 0.349 & 0.219 \\
\hline 9. In the last month, how often have you been able to control irritations in your life? & 0.522 & 0.126 \\
\hline 10. In the last month, how often have you felt that you were on top of things? & 0.660 & 0.105 \\
\hline 11. In the last month, how often have you been angered because of things that happened were outside of your control? & 0.331 & 0.238 \\
\hline 12. In the last month, how often have you found yourself thinking about things that you have to accomplish? & 0.085 & 0.622 \\
\hline 13. In the last month, how often have you been able to control the way you spend your time? & 0.606 & 0.118 \\
\hline 14. In the last month, how often have you felt difficulties were piling up so high that you could not overcome them? & 0.277 & 0.479 \\
\hline
\end{tabular}

The principal component method with varimax rotation was applied to extract patterns.

Table 3. Results regarding the validity of the PSS-14, as compared with the clinical assessment of $n=100$ participants of the study

\begin{tabular}{|c|c|c|c|c|c|}
\hline & Kendall's tau-b & $p$ & Mean difference & $\begin{array}{l}\text { Lower limit } \\
\text { of agreement }\end{array}$ & $\begin{array}{l}\text { Upper limit } \\
\text { of agreement }\end{array}$ \\
\hline Overall sample, $n=100$ & 0.43 & $<0.001$ & 3.53 & -11.95 & 19.01 \\
\hline \multicolumn{6}{|l|}{ Gender } \\
\hline Males, $n=40$ & 0.40 & 0.001 & 3.31 & -13.47 & 20.08 \\
\hline Females, $n=60$ & 0.46 & $<0.001$ & 3.68 & -11.27 & 18.63 \\
\hline \multicolumn{6}{|l|}{ Age group } \\
\hline$<40$ years old, $n=62$ & 0.48 & $<0.001$ & 1.86 & -13.82 & 16.68 \\
\hline$\geq 40$ years old, $n=38$ & 0.26 & 0.05 & 6.52 & -8.98 & 22.02 \\
\hline \multicolumn{6}{|l|}{ Occupation } \\
\hline Private employees, $n=22$ & 0.51 & 0.003 & 1.83 & -14.36 & 18.02 \\
\hline Public employees, n=63 & 0.44 & $<0.001$ & 4.48 & -10.53 & 19.49 \\
\hline Free lancers, $n=13$ & 0.34 & 0.11 & 2.63 & -14.67 & 19.9 \\
\hline \multicolumn{6}{|c|}{ Participants satisfied with their income } \\
\hline Not satisfied, $n=63$ & 0.37 & 0.001 & 2.62 & -14.27 & 19.52 \\
\hline Satisfied, n=37 & 0.58 & $<0.001$ & 5.02 & -6.88 & 16.92 \\
\hline \multicolumn{6}{|l|}{ Family Status } \\
\hline Married, $n=51$ & 0.50 & $<0.001$ & 4.70 & -10.41 & 19.81 \\
\hline Non married, $n=41$ & 0.36 & 0.003 & 1.51 & -13.46 & 16.48 \\
\hline \multicolumn{6}{|l|}{ Having children } \\
\hline No, $n=47$ & 0.44 & $<0.001$ & 1.70 & -13.58 & 16.98 \\
\hline Yes, $n=53$ & 0.42 & $<0.001$ & 5.56 & -9.53 & 20.65 \\
\hline
\end{tabular}

that the structure of these factors were similar to the one reported by the inventors, Cohen and Williamson (18) as well as by other validation studies (see below). The level of agreement between the tool and the "gold-standard" approach was moderate to good, whereas the bias as derived through the Bland \& Altman method was favourably close to zero; however, the limits of agreement 
were relatively high, reflecting a wide dispersion of the differences between the tool and "gold-standard" method. Despite the relatively small sample size, further analyses showed that the Greek PSS-14 was also valid in various subgroups of the studied sample, like men/women, under or over 40 , for those satisfied or not with their income, for married or non-married people, for those who had children or not, and for private or civil service employees. However, the latter finding is limited by the relatively small number of individuals in each sub-group. Moreover, the mean values of the scale were roughly 24/56 in men and 26/56 in women. Current results seem to be in accordance with other reports derived from relevant studies. In particular, using the Chinese version of PSS-14, the mean score of the scale among middle aged men and women was around 23 (out of a theoretical total 56 score) (19). Furthermore, in a study of 1,320 Japanese students, the mean PSS mean score was around 28, using the Japanese version of the PSS-14 (20). As regards European populations, the Spanish PSS-14 mean score among 440 young men and women was around 25 , whereas in a sample of Hungarian adults PSS-14 mean score was quite similar, i.e. $25.3(21-23)$.

The validity of the Greek version of the PSS -14 was also supported by the strong coherence found in all subgroups of tested participants. The latter suggests that PSS-14 can be seen as a reliable scale for the Greek population to use as - thanks to its internal reliability - it seems to measure effectively a distinct set of a construct termed "perceived stress". The aforementioned results are in line with other studies conducted in Europe and in the Asian region, where the values of Cronbach's alpha were similar to the ones presented here.

Furthermore, the majority of studies conducted at acculturating and validating PSS have used a variety of psychological scales and questionnaires as standards and have concluded that PSS is an instrument with good psychometric properties and can be used in either clinical or research settings to estimate chronic stress as a risk factor for diverse psychological symptoms. As it can be seen in Table 3, psychological assessment appears to overestimate the stress levels in general, except for people younger than 40 years of age, those who have no children, those who are private employees, and those who are not married. As mentioned above, PSS -14 estimates stress based on what a person perceives stressful in his environment, on the unique stress response of an individual and the effectiveness of his coping mechanisms, if any. The discordance found to a certain extent between the psychological assessment and the PSS-14 assessment of stress may indicate existence of other factors not measured by PSS-14 but relative to a stress response, which in all likelihood a psychologist would be able to discern, such as the avoidance of someone to admit existence of a problem and the need to explore means of coping with it. Furthermore, use of self-report scales carries certain limitations (24-26). Unfortunately, according to the research of the current study, there are no other published studies in Greece using clinical assessment as a means of validating PSS-14, thus there are no similar findings to be compared. However, it should be noted here that one of the most interesting legitimacy in stress research is the profound disassociation between the psychological and the bodily stress response. In other words, while some individuals perceive high psychological stress without emitting a peripheral physiological stress response, others show strong physiological arousal without perceiving stress. This lack of covariance may be attributed to the complexity of genetic and developmental factors which are implicated in the stress response (12). Moreover, the stress response and its variations shape an additional confounding factor which is decisive in the absence of covariance. It is suggested that there are three response types which serve the organism to cope with a stressor, the immediate stress response that allows the organism to quickly emit a fight or flight response in the face of a stressor; the adaptive stress response which represents the adaptation to secondary signals from the body and the brain; and the evaluative stress response based on cortical structures and pathways involving cognitive processes by which a subject responds to a stressor.

As presented in Table 3, PSS-14 seems to have a lower level of internal consistency for people aged 40 or older, for those not being satisfied with their income, males and public employees. It has been documented before, that women express relatively higher levels of perceived stress than men; this finding has been related to different (i.e. maladaptive) coping styles of women regarding stressful events (26). It has also been reported that stressful appraisals tend to decline as age increases, implying that older people may choose more adaptive coping strategies, even escapism; although, age has been negatively associated with perceived control of one's environment (27). Those employed in the public sector may also express lower levels of perceived stress in relation to private enterprise employees and free lancers, where employment is not so secure or permanent and demands usually exceed the existing resources of the individual, a phenomenon commonly described as work stress. In the present work, it is hypothesized that males, people aged $\geq 40$ and public employees are characterized by relatively lower levels of self reported perceived stress. It is, therefore, indicated that the Greek PSS-14 measures more precisely higher levels of expressed perceived stress and, thus, it seems more internally consistent in specific subgroups. This is because PSS-14 may underestimate perceived stress in subgroups considered less stressed. Moreover, satisfaction with personal income could probably act as a shield regarding levels of perceived stress, since economic strain is in fact a remarkable stressor (28). However, PSS-14 was found to have lower level of internal consistency among people financially unsatisfied, who would otherwise be considered to be under more stress.

Use of PSS-14 has been initially proposed to examine the role of non-specific appraised stress in the etiology of disease and behavioural disorders and as an outcome measure of experienced levels of stress. Furthermore, it has been suggested that PSS -14 can be used to closely evaluate the process by which various moderators of the objective stressor-pathology relationship operate, namely, factors that can alter the stress appraisal and mediate the disease outcome. Given the above, it may be surmised that the present Greek version of PSS-14 can be used for all the aforesaid purposes. The current work provides a valid tool that measures perceived stress to be available in Greek research settings. Use of a valid version of PSS-14 is presumed to further expand research regarding the relationship between perceived chronic stress and various health outcomes in Greece and the comparison of future findings with others from different populations. 


\section{Limitations}

A limitation of the current work is absence of data regarding the educational status of participants, a factor that would further support the sensitivity analysis. Regarding representativeness of the study sample as concerns the whole Greek population, an effort was made to include participants from all age categories (i.e. young, middle-aged and elderly), and both sexes. However, recruitment took place mostly at work place, which included private and public companies; thus, the analysis could not include unemployed people. Moreover, although adequate since the number of $n=100$ participants provided enough power to test validity of the tool, interpretation of the sub-group analyses should be made with caution. Further sampling in various subgroups of the total population, i.e. larger samples of unemployed and retired individuals, having or not children, educated or not people, males and females as well as participants from rural and urban areas would increase the applicability of the tool. Moreover, multiple testing inflated the type-I error. Thus, the robustness of the findings should be targeted on the total sample and not on the sub-groups. Further convergent validity was not tested here since no other valid tool of perceived stress was used. Additionally, the clinical - psychological assessment conducted may have allowed room for information biases and misclassifications diffuse the study.

\section{CONCLUSION}

The Greek version of PSS-14 represents a reliable and valid instrument to use in research settings as a means of generating hypotheses, and can be used to primarily estimate levels of perceived stress as a risk factor of several health issues and behaviours, especially in Greek adult individuals living in urban regions. As far as its elaborative validity is concerned, Greek PSS-14 seems to underestimate levels of stress found by the clinical assessment. Latent traits or constructs should probably be examined and discussed.

\section{Acknowledgement}

We are, particularly, grateful to the men and women from metropolitan Athens and Larissa who participated in and collaborated on this research.

\section{Conflict of interest}

None declared

\section{REFERENCES}

1. Chrousos GP. Stress and disorders of the stress system. Nat Rev Endocrinol. 2009 Jul;5(7):374-81.

2. Hellhammer DH, Hellhammer J. Key issues in mental health. In: Hellhammer DH, Hellhammer J, editors. Stress: the brain-body connection. Basel: Karger; 2008. p. 1-10.

3. McEwen BS, Gianaros PJ. Central role of the brain in stress and adaptation: links to socioeconomic status, health, and disease. Ann N Y Acad Sci. 2010 Feb;1186:190-222.

4. Cohen S, Janicki-Deverts D, Miller GE. Psychological stress and disease. JAMA. 2007 Oct 10;298(14):1685-7.

5. Rosmond R. Role of stress in the pathogenesis of the metabolic syndrome. Psychoneuroendocrinology. 2005 Jan;30(1):1-10.

6. Lupien SJ, de Leon M, de Santi S, Convit A, Tarshish C, Nair NP, et al. Cortisol levels during human aging predict hippocampal atrophy and memory deficits. Nat Neurosci. 1998 May;1(1):69-73. Erratum in: Nat Neurosci. 1998 Aug;1(4):329.

7. Mönnikes H, Tebbe JJ, Hildebrandt M, Arck P, Osmanoglou E, Rose M, et al. Role of stress in functional gastrointestinal disorders. Evidence for stress-induced alterations in gastrointestinal motility and sensitivity. Dig Dis. 2001;19(3):201-11.

8. McBeth J, Silman AJ, Gupta A, Chiu YH, Ray D, Morriss R, et al. Moderation of psychosocial risk factors through dysfunction of the hypothalamic-pituitary-adrenal stress axis in the onset of chronic widespread musculoskeletal pain: findings of a population-based prospective cohort study. Arthritis Rheum. 2007 Jan;56(1):360-71.

9. Torres SJ, Nowson CA. Relationship between stress, eating behavior, and obesity. Nutrition. 2007 Nov-Dec;23(11-12):887-94.

10. Hobfoll SE. Conservation of resources. A new attempt at conceptualizing stress. Am Psychol. 1989 Mar;44(3):513-24.

11. DeLongis A, Folkman S, Lazarus RS. The impact of daily stress on health and mood: psychological and social resources as mediators. J Pers Soc Psychol. 1988 Mar;54(3):486-95.

12. Gunnar M, Quevedo K. The neurobiology of stress and development. Annu Rev Psychol. 2007;58:145-73.

13. Goh C, Agius M. The stress-vulnerability model how does stress impact on mental illness at the level of the brain and what are the consequences? Psychiatr Danub. 2010 Jun;22(2):198-202.

14. Cohen S, Kamarck T, Mermelstein R. A global measure of perceived stress. J Health Soc Behav. 1983 Dec;24(4):385-96.

15. Panagiotakos DB, Pitsavos C, Chrysohoou C, Skoumas I, Stefanadis C; ATTICA Study. Five-year incidence of cardiovascular disease and its predictors in Greece: the ATTICA study. Vasc Med. 2008;13(2):113-21.

16. Madianos MG, Gefou-Madianou D, Stefanis CN. Symptoms of depression, suicidal behaviour and use of substances in Greece: a nationwide general population survey. Acta Psychiatr Scand. 1994 Mar;89(3):159-66.

17. Minas M, Koukosias N, Zintzaras E, Kostikas K, Gourgoulianis KI. Prevalence of chronic diseases and morbidity in primary health care in central Greece: an epidemiological study. BMC Health Serv Res. 2010 Aug 28;10:252.

18. Cohen S, Williamson G. Perceived stress in a probability sample of the U.S. In: Spacapam S, Oskamp S, editors. The social psychology of health: Claremont Symposium on Applied Social Psychology. Newbury Park, CA: Sage; 1988. p. 31-67.

19. Leung DY, Lam TH, Chan SS. Three versions of Perceived Stress Scale: validation in a sample of Chinese cardiac patients who smoke. BMC Public Health. 2010 Aug 25;10:513.

20. Mimura C, Griffiths P. A Japanese version of the Perceived Stress Scale: cross-cultural translation and equivalence assessment. BMC Psychiatry. 2008 Sep 30;8:85.

21. Remor E. Psychometric properties of a European Spanish version of the Perceived Stress Scale (PSS). Span J Psychol. 2006 May;9(1):86-93.

22. Wongpakaran N, Wongpakaran T. The Thai version of the PSS-10: An Investigation of its psychometric properties. Biopsychosoc Med. 2010 Jun 12;4:6.

23. Stauder A, Konkolÿ Thege B. Summary on the validity study of the Hungarian version of the Perceived Stress Scale (PSS) [Internet]. Budapest: Semmelweis University; 2006 [cited 2012 Apr 24];7:203-16. Available from: http://www.psy.cmu.edu/ scohen/PSS_Hungarian_validity_info. pdf.

24. Foster SL, Cone JD. Validity issues in clinical assessment. Psychol Assess. 1995;7(3):248-60.

25. Brummett BH, Babyak MA, Mark DB, Clapp-Channing NE, Siegler IC, Barefoot JC. Prospective study of perceived stress in cardiac patients. Ann Behav Med. 2004 Feb;27(1):22-30.

26. Fountoulakis KN, Iacovides A, Kleanthous S, Samolis S, Gougoulias K, St Kaprinis G, et al. The Greek translation of the symptoms rating scale for depression and anxiety: preliminary results of the validation study. BMC Psychiatry. 2003 Dec 10;3:21.

27. Aldwin CM. Does age affect the stress and coping process? Implications of age differences in perceived control. J Gerontol. 1991 Jul;46(4):P174-80.

28. Pearlin LI, Schieman S, Fazio EM, Meersman SC. Stress, health, and the life course: some conceptual perspectives. J Health Soc Behav. 2005 Jun;46(2):205-19. 